\title{
THE ROLE OF BINARIES IN THE DYNAMICAL EVOLUTION OF THE CORE OF A GLOBULAR CLUSTER
}

\author{
PIET HUT \\ Institute for Advanced Study, Princeton, NJ 08540, U.S.A.
}

\begin{abstract}
.
The size of the core is one of the main diagnostics of the evolutionary state of a globular cluster. Much has been learned over the last few years about the behavior of the core radius during and after core collapse, under a variety of different conditions related to the presence or absence of large numbers of binaries. An overview is presented of the basic physical principles that can be used to estimate the core radius. Four different situations are discussed, and expressions are presented for the ratio $r_{c} / r_{h}$ of core radius to half mass radius. The regimes are: deep collapse in the absence of primordial binaries; steady post-collapse evolution after primordial binaries have been burned up; chaotic post-collapse evolution under the same conditions; and post-collapse evolution in the presence of primordial binaries. In addition, modifications to all of these cases are indicated for the more realistic situation where effects of the galactic tidal field are taken into account.
\end{abstract}

\section{Introduction}

Core collapse is the most dramatic phenomenon in the evolution of globular clusters. It corresponds to the pre-main-sequence stage in stellar evolution, when the energy losses from the photosphere are not yet balanced by nuclear energy generation in the center. In contrast, during the post-collapse evolution of a star cluster some form of central energy source turns on, which makes up for the energy lost through conduction and evaporation of stars from the central regions (for a review of different mechanisms, see Goodman 1993). 
The details of the halt of core collapse only emerged some dozen years ago, and were reported in IAU symposium 113 (Goodman \& Hut, 1985). A few years later, the plot thickened again through the discovery that a significant fraction of stars in globular clusters were contained in binaries. These primordial binaries modify the picture of core collapse considerably. Rather than proceeding down to the miniscule core and enormous densities required in the earlier single-star models, core collapse is halted at moderate central densities of order $\rho_{c} \sim 10^{6} M_{\odot} \mathrm{pc}^{-3}$, and a typical core radius of $r_{c} \sim 0.1 \mathrm{pc}$. The corresponding core contains a few $\times 10^{3} M_{\odot}$, roughly one percent of the inner half mass of the cluster enclosed in a radius $r_{h} \sim 10 \mathrm{pc}$ (Goodman \& Hut, 1989).

A few years ago, we have given a detailed review of primordial binaries in globular clusters, and their effects (Hut et al. 1992). I refer to this paper for many original references, as well as summaries of the history of this field. Many of the more recent theoretical developments concerning the role of binaries in star clusters are discussed by others in these proceedings, e.g. in the contributions by Aarseth, Clarke, Heggie et al., Kiseleva, Leonard, Mardling, McMillan, Phinney, and Rasio \& Heggie. In order to avoid too much overlap with these other papers, I will concentrate here on a single question that is perhaps most relevant to observations: how to estimate the core size $r_{c}$ of a post-collapse cluster. In four separate situations, I will describe the basic physics from which the ratio $r_{c} / r_{h}$ can be estimated, where $r_{h}$ is the half-mass radius. These are given in $\S \S 2-5$ below. $\S 6$ discusses the consequences of adding a galactic tidal field, and $\S 7$ sums up.

\section{Deep Single-Star Core Collapse}

In the late stages of core collapse, the core dynamically decouples from the bulk of the cluster, in a process called 'gravothermal catastrophe' (LyndenBell \& Wood 1968; Spitzer 1987). The reason for this instability is the fact that dynamical evolution proceeds on a relaxation time scale. The relaxation rate is proportional to the number of encounters per star, which in turn is proportional to the density, which is far higher in the core of an evolved cluster than in the bulk of the cluster. Therefore, when the core is losing energy by local 'evaporation' of stars into the surrounding regions, as well as by heat conduction into those regions, the rest of the material has no time to keep up with the shrinking core. Collapse will occur, leading to an infinite density in finite time, unless some other physical process will switch on.

Let us make a rough estimate of the critical size for the core of a star cluster, when core collapse is halted. In the equal-mass point-mass approximation, heat production is proportional to the rate of binary formation in 
three-body encounters, a process that is proportional to the third power of the density:

$$
\dot{E}_{+}=C_{3} \rho^{3} \text {. }
$$

Energy losses, caused by two-body relaxation, are proportional to the number of encounters per unit volume, which in turn is proportional to the square of the density $\rho$ :

$$
\dot{E}_{-}=C_{2} \rho^{2} .
$$

A natural local choice of physical units is one in which the gravitational constant $G$, the mass of a single star $m$, and the root-mean-square velocity of the single stars $v$ are all set equal to unity: $G=m=v=1$. Since the core is close to being self-gravitating, we can use the virial theorem:

$$
N_{c}\left(\frac{1}{2} m v^{2}\right)=\frac{G\left(m N_{c}\right)^{2}}{4 r_{c}}
$$

where $N_{c}$ is the number of particles in the core and $r_{c}$ the core size. We thus find $N_{c}=2 r_{c}$, and $\rho=N /\left((4 / 3) \pi r_{c}^{3}\right) \simeq 2 N^{-2}$. Requiring now that $\dot{E}_{+}=\dot{E}_{-}$, we find

$$
N_{c}=\sqrt{\frac{2 C_{3}}{C_{2}}}=\sqrt{\frac{20}{0.003}} \simeq 80,
$$

where the estimates for the numerical values for the constants are taken from Hut \& Inagaki (1985). A similar estimate leading to $N_{c} \simeq 50$ was made by Goodman (1984). It is clear that deep core collapse leads to the momentary appearance of a tiny core with less than one hundred stars, with $r_{c} \ll 0.01 \mathrm{pc}$ corresponding to a huge density, of order $10^{9} M_{\odot} \mathrm{pc}^{-3}$, for typical globular cluster parameters.

\section{Single-Star Post-Collapse Evolution: Smooth Core Reexpan- sion}

Long after core collapse, on a time scale of several half-mass relaxation times, the outer regions of the cluster are finally able to catch up with what has happened in the center. For small to moderate numbers of stars ( $N \lesssim 10^{4}$; Goodman 1987), the core is able to adjust itself to the demands of these outer regions. Given the rate of energy loss from these regions, the core will find an equilibrium size and corresponding density such that it will form new binaries at the correct rate to make up for the outer energy losses.

Goodman (1984) has made a detailed analysis of this situation, and derived a relation $N_{c} \propto N^{1 / 3}$, which implies $r_{c} / r_{h} \propto N^{-2 / 3}$. For realistic globular cluster parameters, he found $N_{c} \sim 3 \times 10^{2}$, six times larger than 
the deep collapse figure of $N_{c} \sim 50$ that he found. The reason that the core radius becomes about six times smaller during deep collapse, compared to subsequent smooth expansion, is that the latter takes place in nearisothermal equilibrium. As a result, the conduction rate of energy through two-body relaxation is much lower than during the original collapse, which leaves a more substantial temperature gradient in its wake. Lowering the conduction rate is equivalent to lowering the value $C_{2}$, which has the effect of increasing $N_{c}$, according to eq. 4 .

\section{Single-Star Post-Collapse Evolution: Chaotic Core Reexpan- sion}

For large numbers of stars $\left(N \gg 10^{4}\right)$, post-collapse expansion of the core of a star cluster does not proceed smoothly. As discovered by Sugimoto and Bettwieser (1983; Bettwieser and Sugimoto 1984), chaotic fluctuations occur in the size of the core radius. These can be explained as a consequence of the gravothermal instability, and were therefore called 'gravothermal oscillations'.

The underlying physical mechanism can be characterized as follows. For a large number of stars in the system, the inner relaxation timescale is much larger than the half-mass relaxation timescale, which determines the overall rate of expansion. Therefore, the inner regions have the tendency to evolve on a timescale much smaller than the bulk expansion timescale. As a result, the inner regions tend to get impatient, and a small fluctuation can trigger a local re-collapse, followed by a local re-expansion. The larger the number of stars, the more the central and outer timescales are decoupled, and the more chaotic the oscillations become. The dynamical behavior of these oscillations can be shown to be characterized by a low-dimensional chaotic attractor (Breeden et al. 1990; Cohn et al. 1991; Breeden \& Cohn 1995). The gravothermal character of the core oscillations was confirmed explicitly by Goodman (1987), who performed a linear stability analysis of a new regular self-similar model for post-collapse evolution, and classified the different modes of behavior according to the type of linear instability they exhibit.

Like gravothermal collapse, gravothermal oscillations appear to be a ubiquitous phenomenon, at least in the models which treat the stars and all physical processes as continuous quantities. Inagaki (1986) and McMillan $(1986,1989)$ have expressed doubts as to whether the oscillations persist in real clusters, where the stars and the physical processes are discrete, and statistical fluctuations may be large. This issue has now been resolved by direct N-body simulations of systems containing $>10^{4}$ stars, and the conclusion is that oscillations do occur, and indeed have a clearly gravothermal 
nature (Makino, this volume).

Although the details of the gravothermal oscillations are strongly dependent on the total number of stars, as well as their mass spectrum, the maximum value of the core radius is relatively insensitive to those details. Since the evolution slows down most around the time of maximum expansion, observations of a cluster core will find the core to be near maximum expansion in the overwhelming majority of cases (just as a binary star in a very eccentric orbit will almost always have a separation close to twice the semimajor axis). Typical values, from Fokker-Planck calculations including a mass spectrum, are $r_{c} / r_{h} \sim 10^{-2}$ (Murphy et al. 1990).

\section{Post-Collapse Evolution with Primordial Binaries}

Among globular cluster stars, a fair fraction are born as members of binaries. The fraction may not be as large as that in the galactic disk, but it is large enough to have a significant dynamical effect (see Hut et al. 1992 for a detailed review and references). The overall cluster binary population is conveniently parametrized by the binary fraction $f_{B}$, defined as the number of "objects" in a cluster that are actually binaries (so, if binary components are representative of the cluster as a whole, the binary mass fraction is $\left.\sim 2 f_{B} /\left[1+f_{B}\right]\right)$. Because of the presence of many observational selection effects, this quantity is not known accurately, but it probably lies in the range $3-30 \%$, and a value of $f_{B} \sim 10 \%$ is widely taken to be "typical."

The presence of primordial binaries radically changes the picture sketched above, in which clusters undergo deep core collapse, before re-expanding, with or without core oscillations. As was pointed out by Goodman \& Hut (1989), gravitational 'burning' of binaries will cause the core collapse to be halted at a far larger core radius of $r_{c} \approx 0.02 r_{h}$, compared to $r_{c} \sim 10^{-4} r_{h}$ for deep core collapse.

This phenomenon has its analogue in stellar evolution, where a protostar does not land directly on the hydrogen main sequence, but instead spends a brief time on the deuterium main sequence. While primordial deuterium is being burned in the center of the star, its radius remains significantly larger than its eventual value. The reason is the high efficiency of deuterium burning at relatively low temperature, compared to hydrogen burning. As a result, central heat production and surface heat loss are balanced at a lower central temperature, and hence larger stellar radius.

Similarly, the high efficiency of gravitational energy extraction from an existing binary population, compared to binary production from single stars only, allows the cluster core to remain relatively large, until the primordial binaries have been depleted. Numerical conformation of the semiquantitative estimate given above, for $N$-body simulations with $N \leq 2 \times 10^{3}$ 
(McMillan et al. 1990, 1991; McMillan and Hut 1994) showed that for $N=(1 \sim 2) \times 10^{3}$, the core radius $r_{c} \approx(0.10 \sim 0.15) r_{h}$. This is larger than the value predicted by Goodman and Hut (for this $N$ range, their eq. (6) would give $r_{c} \approx 0.05 r_{h}$ ). Most likely, this discrepancy is caused by the low $N$ values used in direct $N$-body simulations so far; it is not clear whether values of $N \sim 10^{3}$, with total numbers of core stars $N_{c}<10^{2}$, are high enough to reach the asymptotic scaling regime. Fokker-Planck calculations by Gao et al. (1991) resulted in values in the range $r_{c} \approx(0.01 \sim 0.04) r_{h}$, in broad agreement with the earlier analytical estimate. A more definite answer will soon be provided, when we will run primordial binary simulations on the GRAPE-4, for $N \geq 2 \times 10^{4}$.

\section{Effects of the Galactic Tidal Field on Post-Collapse Evolution}

Let us now take into account the fact that globular clusters do not evolve in isolation, but are tidally limited by the presence of the gravitational field of the galaxy. This means that the average density of the cluster is forced to remain at a fixed value, comparable to the average density of the matter inside its orbit (for a circular orbit; the situation is qualitatively similar for orbits of moderate eccentricity). As a result, $r_{h}$ is forced to shrink upon mass loss through evaporation of cluster stars, in stark contrast to the evolution of isolated clusters, for which $r_{h}$ grows steadily after core collapse.

Since mass segregation tends to concentrate the heavier binaries towards the core, most of the evaporating stars are single. Therefore, the depletion of binaries in the core by gravitational 'burning' may or may not be offset by the depletion of single stars through evaporation from the outer parts of the cluster. For each cluster, with a given mass and size, there is a critical binary fraction $f_{B, c r i t}$, forming a watershed between these two possibilities. For $f_{B}<f_{B, \text { crit }}$, primordial binaries will be burned up before the cluster as a whole dissolves in the tidal background field. In this case, the later stages of cluster evolution will show gravothermal oscillations (if $N$ is large enough). However, for $f_{B}>f_{B, c r i t}$, primordial binaries will remain present in the core until the end, and the overall binary fraction $f_{B}$ will in fact increase in the later stages of the cluster. Gravothermal oscillations will not occur during any stage of cluster evolution, in this case.

These two possibilities are illustrated in fig. 1, based on the simulations reported by McMillan and Hut (1994). The watershed value here is $f_{B, c r i t} \sim$ $15 \%$. The precise value of $f_{B, c r i t}$ shows some dependence on the cluster parameters, especially on the size of the tidal radius $r_{t}$ (for $r_{t}<0.8 r_{h}$, the standard choice in our simulations, $f_{B, \text { crit }}$ will be smaller, since single star evaporation will be more rapid). In practice, $f_{B, c r i t}$ may well be lower than estimated here, if we take tidal shocking processes into account, which 

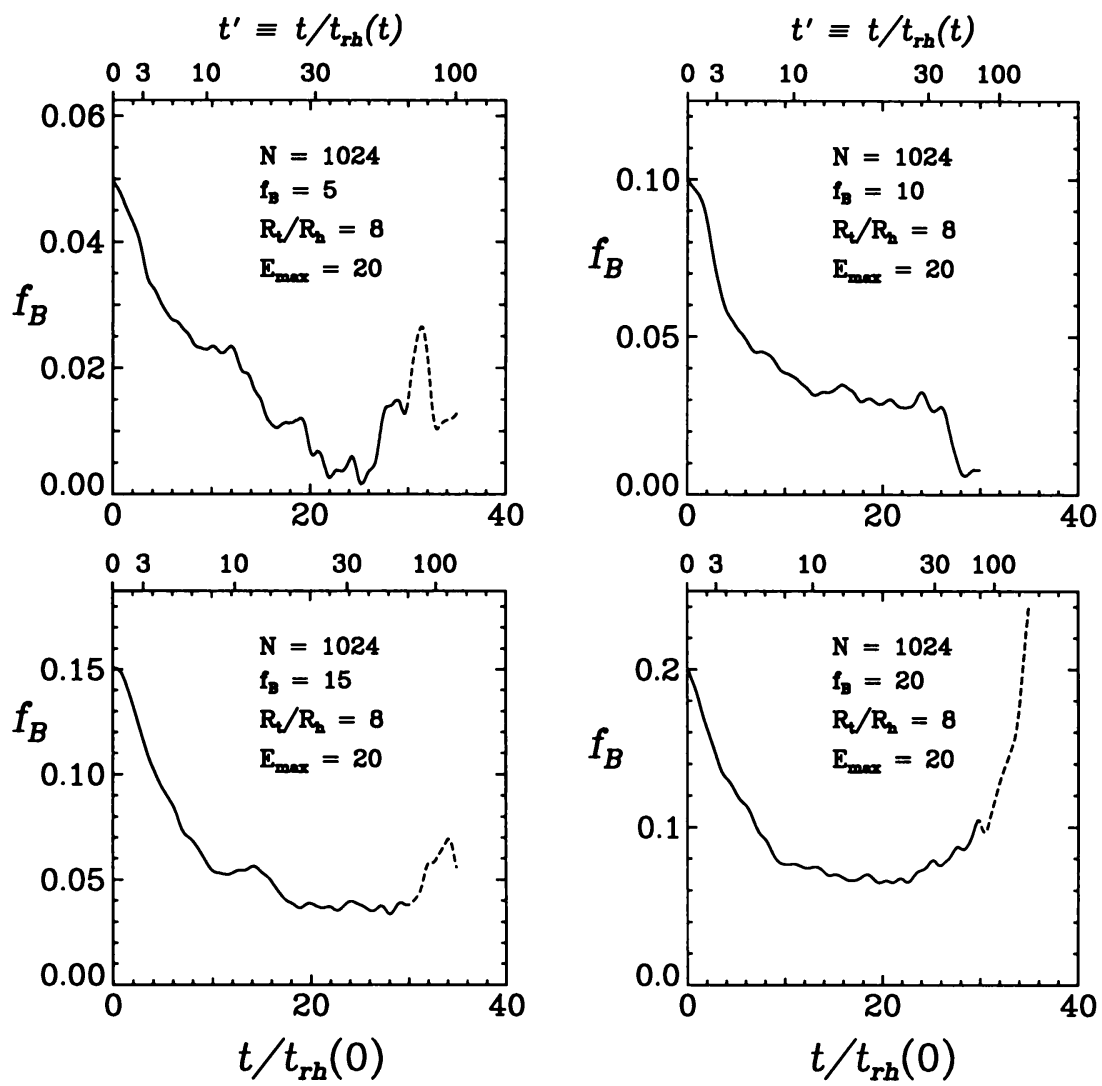

Figure 1. Binary fraction $f_{B}$ as a function of time $t$, in units of the original half-mass relaxation time. $N$ denotes the number of particles in the simulation, $R_{t} / R_{h}$ the ratio between tidal radius and half-mass radius, and $E_{\max }$ the maximum value in the spectrum of initial binding energies of the primordial binaries. The dashed portion of the lines indicate where the total number of stars has dropped to such a low value that low-number statistics make the result unreliable.

recently have been shown to be more efficient than previously estimated ( $c f$. Weinberg 1994; Kundić \& Ostriker 1995).

In fig. 2, the core population of binaries is followed. Plotted is $f_{B c}$, the fraction of objects in the core that are binaries, rather than single stars or multiple star systems containing more than two stars. Again, it is clear that for $f_{B}<15 \%$ the core binary population will eventually drop to very low values, whereas for $f_{B}>15 \%$ the core binary population will remain constant, within the numerical noise. The fraction of the cluster mass that is present in the core will increase towards the end of the lifetime of the cluster, as the outer regions are eaten away by the galactic tidal field (see McMillan and Hut 1994 for a detailed discussion). For values of $f_{B}$ above 

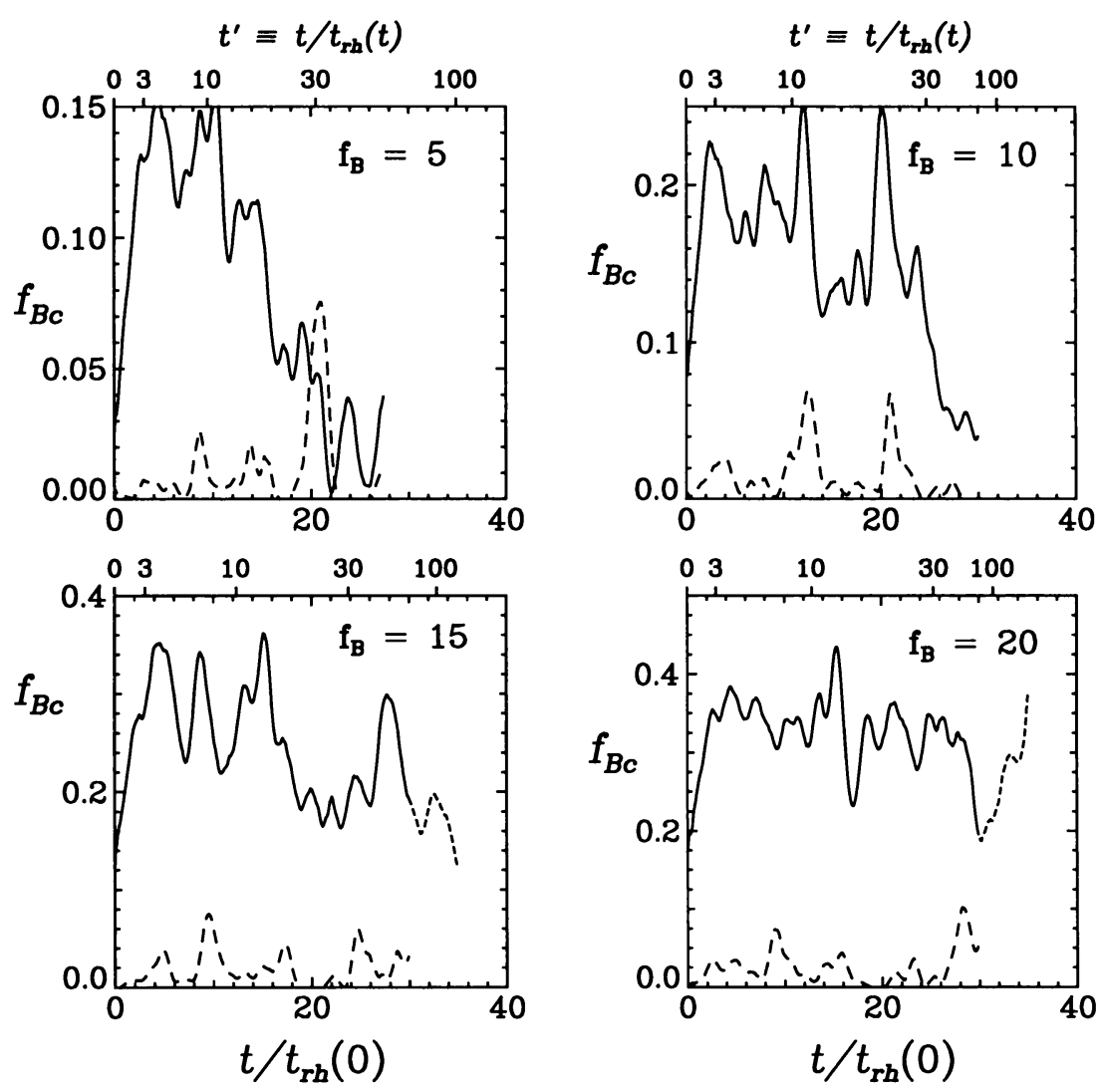

Figure 2. Binary fraction $f_{B c}$ in the core, as a function of time. $f_{B}$ denotes the initial value of the overall binary fraction. The long-dashed line indicates the fraction of triple stars.

the watershed, this implies a growing core size, in units of the half mass radius, as is evident in Fig. 3. For smaller values of $f_{B}$, however, there is a near-cancellation of two tendencies, resulting in a near-constant value for $r_{c} / r_{h}$. An isolated cluster would show a shrinking core (fewer binaries lead to a higher density required for the same energy generation rate, fixed by the rate of energy loss at this outskirts), and an expanding half-mass radius. Both effects would lead to a decrease in $r_{c} / r_{h}$. A tidally truncated cluster, however, would show a decrease in $r_{h}$ as well as a decrease in $r_{c}$. In the case of the present simulations, these two effects turn out to be comparable in magnitude. 

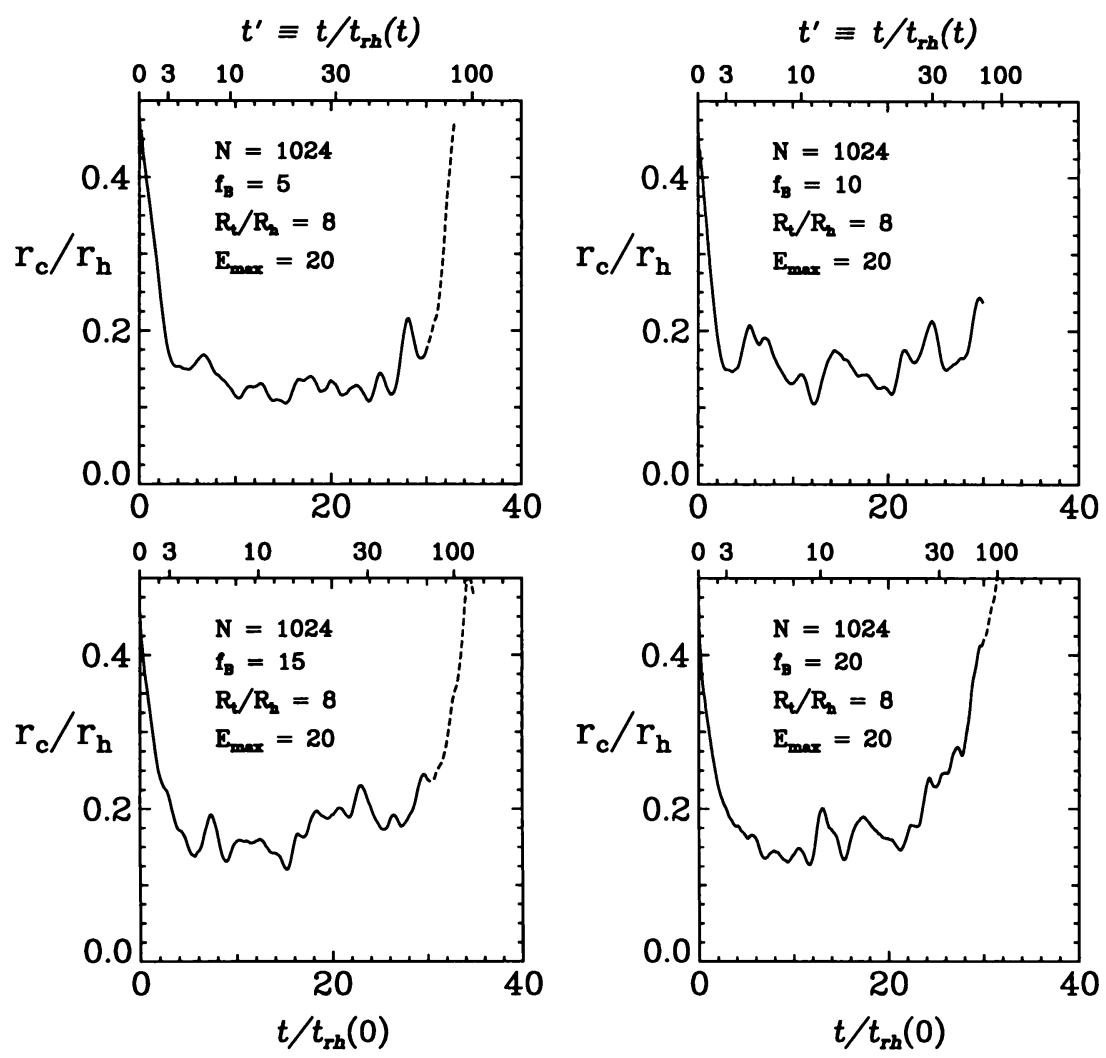

Figure 3. Core radius $r_{c}$ in units of the half mass radius $r_{h}$, as a function of time; labels as in fig. 1 .

\section{Summary and Discussion}

It is quite possible that primordial binaries may provide the only type of fuel needed to power post-collapse evolution, for most globular clusters (McMillan \& Hut 1994). In this case deep core collapse will not occur, and the core will contain a significant fraction of binaries at any time, even though the rest of the cluster may become more depleted in binaries through mass segregation.

For those clusters that do run out of primordial binaries, subsequent post-collapse evolution most likely will show chaotic core oscillations, with relatively large core radii almost all of the time, comparable to the case in which primordial binaries are still present. In both cases, $r_{c} / r_{h} \sim 10^{-2}$, within a factor of a few. The precise value of this factor is crucial for comparison with observations, but has not been determined so far from simulations. Fokker-Plank methods are inherently not well fit to deal with primor- 
dial binaries, and direct $N$-body calculations with primordial binaries have not yet been carried out for sufficiently large $N$ values. Fortunately, the GRAPE-4 computer will be up to the challenge, and is expected to provide us with the answer within a year or so. At that time, we will be able to judge whether the result reported by Guhathakurta (these proceedings), that in M15 the core radius $r_{c}<0.1 \mathrm{pc}$, is compatible with either or both scenarios (primordial binary burning versus gravothermal oscillations).

\section{Acknowledgements}

I thank Steve McMillan for his help in constructing the figures, based upon the runs reported in McMillan and Hut (1994). I thank Profs. Sugimoto and Makino, as well as the whole GRAPE group, for their hospitality during my visit to Tokyo University, when this paper was written. I am also grateful for the grant from JSPS which enabled me to make this visit.

\section{References}

Bettwieser, E. \& Sugimoto, D. 1984, MNRAS, 208, 439

Breeden, J.L., Packard, N.H. \& Cohn, H.N. 1990, preprint

Breeden, J.L. \& Cohn, H.N. 1995, ApJ, 448, 672

Cohn, H.N., Lugger, P.M., Grabhorn, R.P., Breeden, J.L., Packard, N.H., Murphy, B.W. \& Hut, P. 1991, in The Formation and Evolution of Star Clusters, A.S.P Conference Series, 13, ed. K. Janes (ASP, San Francisco), p. 381

Gao, B., Goodman, J., Cohn, H. \& Murphy, B. 1991, ApJ, 370, 567

Goodman, J. 1984, ApJ, 280, 298

Goodman, J. 1987, ApJ, 313, 576

Goodman, J. 1993, in Dynamics of Globular Clusters, eds. S. Djorgovski and G. Meylan (San Francisco: ASP), p. 87

Goodman, J. \& Hut, P. (eds.) 1985, Dynamics of Star Clusters, IAU Symp. No. 113 (Dordrecht: Reidel)

Goodman, J. \& Hut, P. 1989, Nature, 339, 40

Hut, P. \& Inagaki, S., 1985, ApJ, 298, 502

Hut, P., McMillan, S. L. W., Goodman, J. G., Mateo, M., Phinney, E. S., Pryor, C., Richer, H. B., Verbunt, F., \& Weinberg, M. 1992, PASP, 105, 981

Inagaki, S. 1986, PASJ, 38, 853

Kundić, T. \& Ostriker, J. P. 1995, ApJ, 438, 702

Lynden-Bell, D. \& Wood, R 1968, MNRAS, 138, 495

McMillan, S.L.W. 1986, ApJ, 307, 126

McMillan, S.L.W. 1989, in Dynamics of Dense Stellar Systems, ed. D. Merritt (Cambridge University Press), p. 207

McMillan, S.L.W., Hut, P. \& Makino, J. 1990, ApJ, 362, 522

McMillan, S.L.W., Hut, P. \& Makino, J. 1991, ApJ, 372, 111

McMillan, S. \& Hut, P. 1994, ApJ, 427, 793

Murphy, B.W., Cohn, H.N. \& Hut, P. 1990, MNRAS, 245, 335

Spitzer, L., 1987, Dynamical Evolution of Globular Clusters [Princeton Univ. Pr.]

Sugimoto, D. \& Bettwieser, E. 1983, MNRAS, 204, 19p

Weinberg, M. D. 1994, AJ, 108, 1414 